\title{
Method of Top-level Design for Automated Test Systems
}

\author{
Zhenjie Zeng ${ }^{1}$, Xiaofei Zhu ${ }^{1, *}$, Shiju Qi ${ }^{1}$, Kai Wu ${ }^{2}$ and Xiaowei Shen ${ }^{1}$ \\ ${ }^{1}$ Rocket Force University of Engineering, Xi'an, China \\ ${ }^{2}$ Troops No. 96604, Beijing, China \\ ${ }^{*}$ Corresponding author
}

\begin{abstract}
When designing an automatic test system, it is necessary to make each electronic test device conform to different test requirements. The most important issue is the system toplevel design. The article starts with the three steps of the top-level design: system requirements analysis, architecture selection and analysis, and test equipment configuration. It describes in detail how to develop the top-level system design efficiently and reasonably when developing automated test systems. The principles, available method techniques, and precautions have some guiding significance for the top-level design of automated test systems.
\end{abstract}

Keywords-automatic test system; top-level design; requirements analysis; architecture selection; test equipment configuration

\section{INTRODUCTION}

Usually, with a minimum of human involvement, a computer is used to execute a software program to control the test process and perform data processing until the test system that gives the test results in an appropriate manner is called ATS (Automatic Test System) or ATE (Automatic Test Equipment). .

With the advancement of test bus technology, computer technology and software engineering technology, the difficulty of establishing ATS systems is also increasing. Due to the diversification of test objectives, there is no bus that can cover the needs of the entire automated test, coupled with the complexity and diversification of the test process and the function of the test instruments, making the establishment of modern automated test systems, especially the design of test software. The difficulty has doubled. How to effectively and rationally plan the test system architecture and select test equipment is a place that is not yet perfect, and therefore the top level design of the automatic test system is getting more and more attention.

\section{TOP-LEVEL DESIGN}

As the name suggests, the top-level design is the overall planning and design at the highest level. The top-level design of automatic test system integration is to stand at the level of past, present and future demands of the system under test, and to conduct overall planning and design from the perspective of technological development.
The top-level design of automatic test system integration is based on sufficient requirements analysis, and comprehensively considers the optimal matching of technical and economic performances. It is advanced, practical, open, real-time, universal (compatibility), and reliability. , maintainability and other aspects of a comprehensive analysis, determine the test system architecture (including hardware platforms and software platforms), develop a corresponding test program. As shown in Figure 1, it is usually divided into three steps: requirements analysis, architecture selection and analysis, and test equipment configuration.

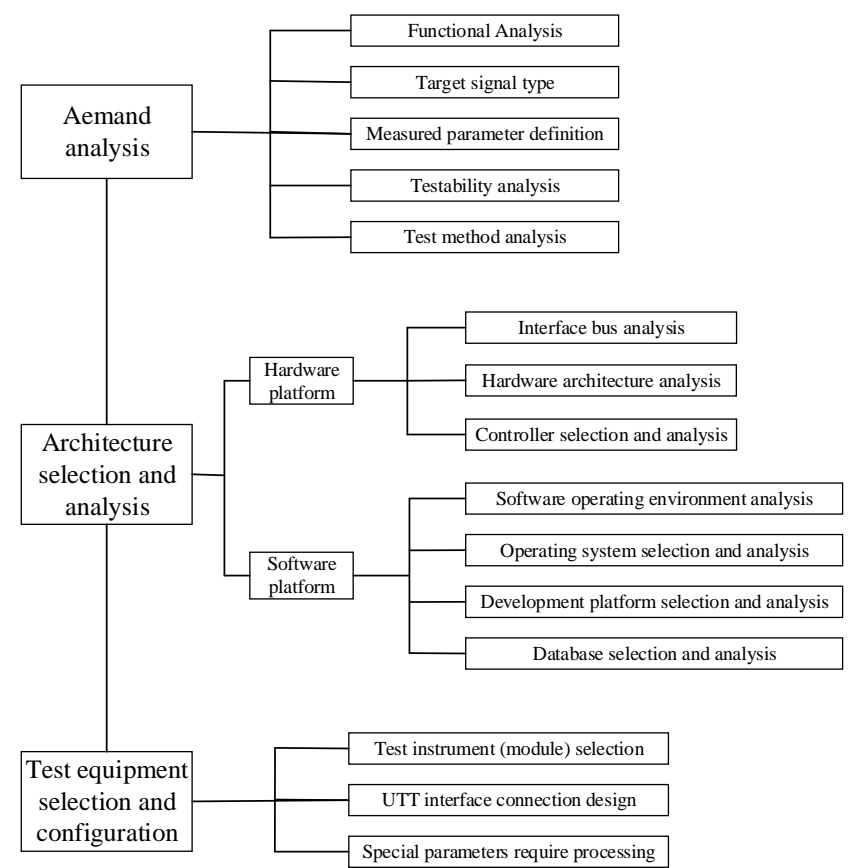

FIGURE I. AUTOMATIC TEST SYSTEM INTEGRATION TOP LEVEL DESIGN FLOW

\section{DEMAND ANALYSIS}

Test requirement analysis is the basis of automatic test system integration top-level design. It mainly contains five aspects: functional requirements of the test target, test parameters, test objects, test methods, and test system planning. 


\section{A. Test Target Functional Requirements}

The different requirements of the test equipment working platform determine the test speed requirements, and also determine the different requirements of the online/offline test; the main control method and logic of the tested equipment determines the difference between the test procedures and methods; the input frequency of the tested equipment, Different parameters, such as amplitude and modulation method, determine the overall requirements for the operating frequency band, small signal level (minimum leakage), and waveform parameters of the automatic test system analog signal source; the output and content of the device under test determines the signal sampling of the automatic test system. The data acquisition method is different; the digital communication interface of the device under test determines that the digital communication interface that the automatic test system should have is different from the protocol; the testability interface of the device under test determines the final test capability and fault diagnosis ability of the automatic test system.

\section{B. Test Parameters}

The test parameter analysis includes analysis: the form of the measured parameter (electrical or non-electrical, digital or analog, etc.), range and quantity; performance index (measurement accuracy and speed, etc.); the form and range of the excitation signal. In particular, when analyzing requirements for a top-level design of a general-purpose comprehensive automatic test system that is suitable for multiple systems, multiple protocols, and multiple equipment, comprehensive analysis is often required to integrate the test parameters.

\section{Test Object}

The test objects vary widely. When analyzing the test objects, a comprehensive analysis must be performed in conjunction with the test system requirements of the test objects. In the face of a specific test object test system or subsystem, the description can use a variety of expressions to give different models of the test system at different levels of simplification, such as language descriptions, graphics, and mathematical formulas. As a simplified description of some test systems, their models merely express their basic characteristics, often ignoring irrelevant details in order to simplify their complexity. For a complex test object test system, a model is inevitably limited by some assumptions in its design and utility. These conditions often have some ambiguity and basically reflect an implicit conceptual idea. Therefore, when analyzing the requirements of a specific test object, it is usually necessary to establish a corresponding test system model.

\section{Test Methods}

According to the functional requirements of the test target, a corresponding test method is formulated for the "face-to-face automatic test system" or "object-oriented automatic test system”..

\section{E. Test System Planning}

When developing an automated test system, it often takes a lot of time to complete the test-assisted tasks such as creating files and programming supporting test software. The test application software development platform can standardize all kinds of test processes and integrate an operating system that is suitable for various test and post-processing functions. It can help us to complete these test auxiliary work; therefore, we use this kind of test platform to conduct various tests. When testing, you can save a lot of time.

\section{ARCHITECTURE SELECTION AND ANALYSIS}

On the basis of sufficient requirements analysis, determining the architecture of the automated test system is the most critical step in the top-level design. That is how to determine the test plan from the perspective of the top-level design, and select the hardware platform and software platform architecture of the automatic test system, and the most important one is the selection of the test equipment digital communication interface bus.

\section{A. System Test Plan Selection}

The system test plan is the overall concept of product testing. It specifies the type of product testing, when (continuous or regular) testing, where (field or workshop, or which maintenance level), testing methods, and test methods used. The types of system test can be divided into: system-wide test and departmental system test, static test and dynamic test, online test and offline test, quantitative test and qualitative test, continuous test and periodic test, etc. The test level can be divided into three levels according to the location: production site, use site, and maintenance base. The test system (equipment) operating methods are generally:

According to the use of the operation can be divided into three kinds of automatic, semi-automatic and artificial; according to the general degree of application can be divided into two kinds of special and general equipment; according to the association with the product can be divided into two kinds of BITE and external test equipment.

Most of the test methods used in automated testing have so far been modeled on manual tests, from the measurement principles used, the testing techniques used, to the test procedures performed, except that computers were used instead of manual operations. As far as the characteristics and potential of automatic testing are concerned, fundamental reforms of the test plan are needed for future research.

\section{B. Selection of Test Equipment Digital Communication Interface Bus and ATS Structure}

The development of automatic test systems has promoted the continuous emergence of various general-purpose test equipment interface buses and rapid technological advancement: from the early GPIB, CAMAC to the recent VXI, MXI, PCI, PCIe, PXI, PXIe, cPCI, MMS, IEEE1394 ( Firewire), USB, etc. Although technical characteristics are not the same, they are widely used. 
The structural elements of a modern automated test system are programmable test instruments, test controllers, interconnected standard digital interfaces, and software systems. At present, modern automatic testing has been widely used, and the test objects faced are large, complex, and diversified, making it impossible for an automatic test system based on any kind of bus technology to cover the needs of the entire test object.

Multi-bus fusion automatic test system structure shown in Figure 2. It consists of test instruments, DUTs(design under test) and UUT(unit under test) interfaces, test controllers (computers), various general-purpose digital interface buses, and test software. The test controller is interconnected with the test instrument through the digital interface bus, and the device under test is connected to the input/output terminal of the test instrument through the UUT interface. The digital interface bus used may be GPIB, VXI, PXI, LXI, or even an internal computer bus (AT/EISA/PCI), or their convergence. Once the standard digital interface bus architecture used is determined, the automatic test system architecture is basically selected. In an automatic test system, regardless of the interface bus architecture, an external computer or built-in computer system can be selected as the test system controller. The choice of the test system controller should fully consider the optimal matching of technical and economic performance, and choose from real-time, practical, reliable, flexible and convenient.

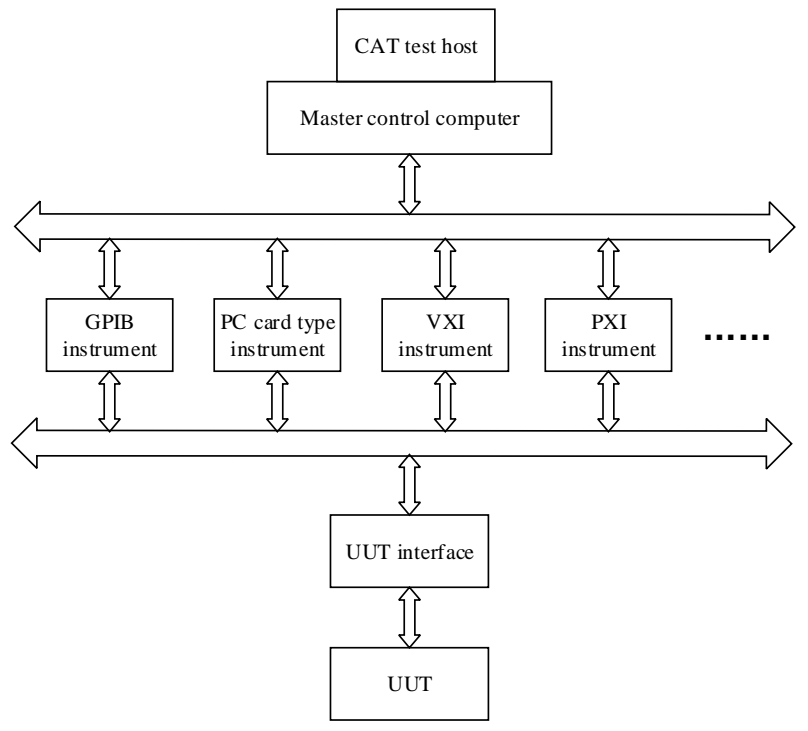

FIGURE II. MULTI-BUS FUSION AUTOMATIC TEST SYSTEM STRUCTURE

\section{Test Software Platform Mode Selection}

In modern computer-based automated test systems, hardware is the foundation and software is the soul. Test software has increasingly become the main body of ATS, which determines the advanced nature, reliability, practicality, and real-time performance of the entire automated test system.

The automatic test software platform mainly refers to the programming language and software support environment involved in the test application software design. It is an integrated software platform such as a computer operating system, a test programming language, a database software, and a program diagnosis software. The key element is Test programming language. Since the automatic test system was popularized and applied, there have been great developments in testing programming languages from low-level to high-level, to the current test application development environment.

\section{TEST EQUIPMENT CONFIGURATION}

After the system structure of the test system is determined, the next task is to synthesize the test contents according to the requirements analysis, and to match the corresponding test equipment according to the test content requirements. There are three types of optional test equipment: general test equipment, special purpose equipment, and test interface adapter.

\section{A. Universal Test Equipment}

The universal test equipment includes a main box, a test controller, a main control interface, a zero slot controller, an instrument module, and a desktop instrument. The following factors should be considered when selecting the type of equipment: (1) The higher the degree of equipment automation, the shorter the time for detecting and isolating faults, and the less the manpower consumption, but the cost of test equipment will increase and more protection is needed. (2) Differences in capabilities between the two are to be considered when selecting a BIT (Built-in-Test) and an off-board automatic test equipment. (3) When the BIT is used in conjunction with the off-board automatic test, make full use of the BIT capability of each unit under test. (4) When selecting a dedicated or generalpurpose device, it is necessary to consider that the specialpurpose device is simple and convenient to use and has high efficiency, but the use range is narrow. (5) The main selection of instrument and equipment is based on the requirements of test parameters, characteristics of the signal to be measured, and range selection. When selecting the instrument module, pay attention to the size of the bus module, power, and number of slots.

\section{B. Special Purpose Equipment}

When the test is not ready for selection, in addition to the above-mentioned common tests, when preparing for the following situations, it may be considered to develop or develop special purpose instrument (module) equipment. When the current product can not meet the test requirements, multiple instruments and equipments are required to complete the measurement together. However, the utilization rate of each instrument is very low or can be accomplished with one instrument. When the price is high and the utilization rate is low, the use of development or development is considered. Special purpose instrument.

\section{Test Interface Adapter Design}

For different test objects, the extraction and feeding of various test signals requires the design and manufacture of various test interfaces and special fixtures. In the automatic test system, especially the automatic test system assembly of complex electronic equipment, the requirements of the same type but different models and different test objects exist 
universally, and often require the test system group to build a relatively universal automatic test platform. Through this platform, different test modules and test methods can be used to quickly and easily complete the automatic test system set-up (configuration) task for different test objects; however, the test interface and the dedicated test module cannot be matched and can only be tested according to the device under test. The test requires the development of a test interface adapter.

\section{CONCLUSION}

This article starts with the three steps of the top-level design: system requirements analysis, architecture selection and analysis, and test equipment configuration. It describes in detail how to perform top-level design efficiently and reasonably when developing automated test systems, and analyzes what the design must follow. Principles, methods, techniques, and precautions have certain guiding significance for the top-level design of automated test systems.

\section{REFERENCES}

[1] LI Xing-shan, ZUO Yi, SUN Jie. Automatic Test System Integration Technology[M]. Publishing House of Electronics Industry, 2004.

[2] QIN Hong-lei, LU Hui et al. Automatic Test System. Beijing: Higher Education Press, 2007

[3] LIU Si-jiu, ZHANG Li-yong. Automatic Test System and Virtual Instrument. Beijing: Publishing House of Electronics Industry, 2009

[4] GU Zhi-yong, TENG Peng, HU Shi-guo, et al. Top-level design of ATS overall plan for integrated helicopter display systems[J]. Electro-optics and Control, 2008, 15(11):59-62.

[5] GU Ya-ping. Research on Top Design of VXI Bus Testing Technology[J]. Electronic Testing, 1998(8):22-23. 